\title{
CAAD EN LA CONSERVACIÓN DE EDIFICIOS HISTÓRICOS.
}

(CAAD IN THE PRESERVATION OF HISTORIC BUILDINGS)

David Kurmann ${ }^{1}$, Moreno Piccolotto ${ }^{1}$, Olga Río ${ }^{2}$, Sarkis Shahinian ${ }^{3}$

(1) Chair of Architecture and CAAD. ETH Zurich

(2) Instituto Eduardo Torroja. CSIC. Madrid

(3) Institute for Building Technologies. ETH Zurich

Fecha de recepción: 6-VI-96 403-18

\section{RESUMEN}

En este articulo se presentan distintas posibilidades del CAAD -Diseño Arquitectónico Asistido por Ordenador- como apoyo a los especialistas involucrados en la conservación de edificios histórico-monumentales. La conservación del patrimonio histórico-artistico es, hoy en dia, tema de interés prioritario a nivel internacional. El CAAD ofrece un rico conjunto de herramientas y métodos mediante los cuales historiadores, arquitectos e ingenieros pueden analizar, a la vez que experimentar, las distintas propuestas de intervención sobre un monumento y visualizar sus postbles consecuencias. La reconstrucción asistida por computador y la simulación del monumento a estudiar pueden facilitar la comprensión de los trabajos a realizar, asi como su impacto antes de la realización de la misma. Por otra parte, las ventajas que presenta el intercambio de datos tanto de texto como gráficos a través de www, permite a los expertos discutir entre si el impacto de las distintas soluciones. Basándose en la propuesta de reconstrucción de un monumento existente, se comentarán diferentes aspectos de las herramientas que aqui se mencionan. Se presentará una herramienta (Sculptor), con la cual se realizaron distintos modelos tipológicos del monumento, a la vez que se mostrarán algunos de los modelos analizados utilizando programas $C A D$ comerciales.

\section{SUMMARY}

In this paper we will discuss the possible contributions of CAAD - Computer Aided Architectural Design - to sustain the efforts of specialists involved in the preservation of historic buildings and monuments. The preservation of the historic and artistic patrimony is nowadays a prior interest at international level. CAAD provides a rich set of different tools and methods with which historians, architects and engineers can analyse, develop and test their interventions on the monument, discuss the consequences of their actions and visualize the effects, enhancing different aspects. Computer-based reconstruction and visualization of the monument of interest can yield important insights before any modification has taken place. Taking advantage of the possibilities offered by the world wide web (www), experts can discuss their results with other experts with textual descriptions, pictorial information as well as complete computer models of varying level of abstraction. Based on the reconstruction proposal of an existing monument, we will discuss different aspects of the tools mentioned herein. We will present a tool (Sculptor), with which we reconstructed various typological models of the monument. Based on this analysis we modelled the modified monument with commercial CAD programs.
Palabras clave: $\mathrm{CAAD}$, realidad virtual, patrimonio arquitectónico, modelos asistidos por ordenador
Key words: CAAD. virtual reality (VR), patrimony, computer aided models 


\section{Introducción}

Existe una gran experiencia en el uso de herramientas $\mathrm{CAAD}$ en diseño arquitectónico. Las aplicaciones usadas en diseño, simulación, modelización y visualización de futuros edificios son innumerables y como se explica en este trabajo pueden resultar también de gran ayuda para aquéllos involucrados en la preservación de edificios histórico-monumentales.

Las herramientas CAAD resultan de gran ayuda para estructurar toda la información de interés recopilada sobre un monumento en estudio y que puede proceder de las mediciones realizadas in situ, de fotogrametría, etc., para ser utilizada en la creación de modelos CAAD, tanto del propio edificio como de su entorno, en la creación de catálogos de documentos sobre el edificio estudiado, para la simulación del proceso constructivo o, simplemente, para visualizar intervenciones previas que se han ejecutado en el monumento, entre otras.

Los dibujos en 2D, las modelizaciones 3D, las técnicas de realidad virtual -VR- [Kalawsky, 1993] y la animación son de gran utilidad para poder hacer una exploración del edificio [Schmitt, 1994] y, a criterio de los autores de este trabajo, para facilitar las labores de los expertos, permitiéndoles tanto crear como visualizar las distintas propuestas.

Los avances recientes de las técnicas multimedia sumados a las distintas herramientas existentes para el dibujo de planos y secciones, la estimación de costos, etc., permiten a los expertos implicados en restauración la creación de aplicaciones sofisticadas que facilitan la documentación del monumento posibilitando, tanto a expertos como a los que no lo son, entender las distintas soluciones al problema.

No obstante, todas estas posibilidades que se han mencionado, y que pueden realizarse utilizando distintas aplicaciones de lo que se consideran herramientas $\mathrm{CAAD}$ tradicionales, adolecen de ciertas limitaciones, a la vez que, no cubren el proceso de diseño completo. Las herramientas $\mathrm{CAAD}$ comerciales son muy útiles cuando se trabaja con valores exactos, o cuando se conocen las dimensiones de los elementos a modelizar, pero no cuando se trabaja con ideas vagas o conceptuales como las que se tienen en las primeras etapas del proceso de diseño, o las etapas donde se elaboran los conceptos para la solución de los distintos problemas. El proceso de abstracción necesario para reducir la complejidad del problema no puede ser tratado utilizando estos programas, lo que conlleva a los expertos a utilizar métodos tradicionales -papel y lápiz- para elaborar las primeras ideas y sólo utilizar el ordenador cuando se tienen suficientes elementos que permitan definir el modelo.
Por último, y no por ello menos importante, el costo de algunas de estas aplicaciones profesionales, así como de los equipos que requieren, pueden constituir un factor crítico para muchos de los potenciales usuarios.

En los siguientes apartados, se presentarán brevemente algunas de las posibilidades que of rece Sculptor, herramienta creada para tratar las fases iniciales del proceso de diseño, así como su utilización para hacer un estudio tipológico de un monumento existente: la capillamausoleo de St. Asvatzatzin en Amaghu Noravank en el cual se plantean distintos tipos de escenarios de restauración.

\section{La herramienta de diseño: Sculptor}

Sculptor es una herramienta para el diseño arquitectónico virtual, desarrollada en los últimos años por D. Kurmann en la ETH Zürich [Kurmann, 1995]. A diferencia de las herramientas CAD existentes, las cuales se utilizan principalmente para generar planos, y por tanto requieren, datos precisos de la construcción, o lo que es lo mismo, son adecuadas en los estadios finales del diseño, Sculptor trata de imitar y modelizar las experiencias humanas siendo, por tanto, muy útil en las primeras etapas del diseño.

Sculptor [Kurmann et al., 1995] trabaja utilizando dispositivos 2D y 3D, tanto de entrada como de salida (ver fig.1), lo que permite la modelización interactiva dentro de un espacio virtual, esto, sumado a la posibilidad de introducir volúmenes positivos y negativos (ver fig. 2) hace que la creación y manipulación de modelos arquitectónicos se pueda hacer de manera fácil.

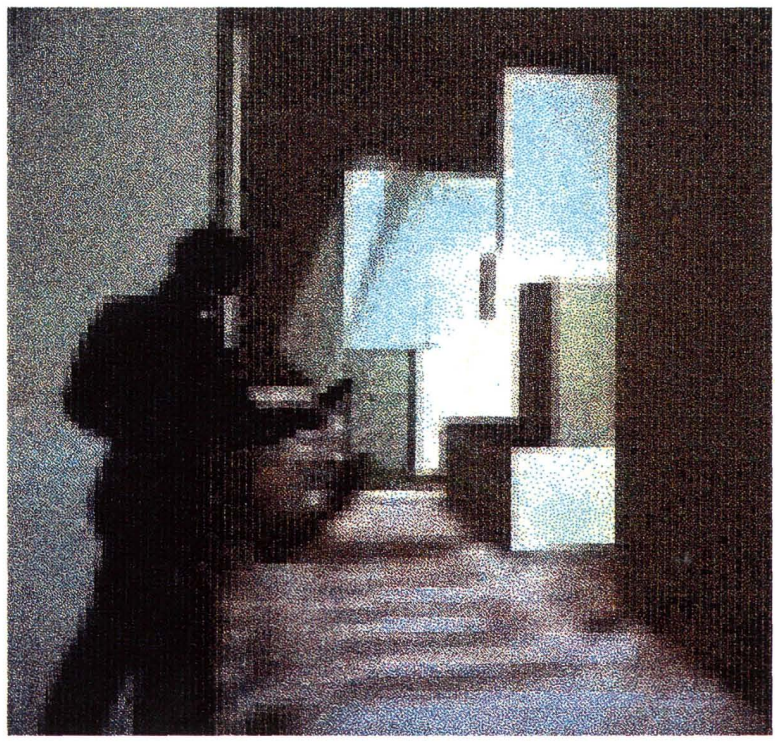

Fig.1.- El usuario utilizando unas gafas 3D se posiciona dentro del propio escenario virtual (proyectado dentro de una gran pantalla utilizando un cañón de proyección) 


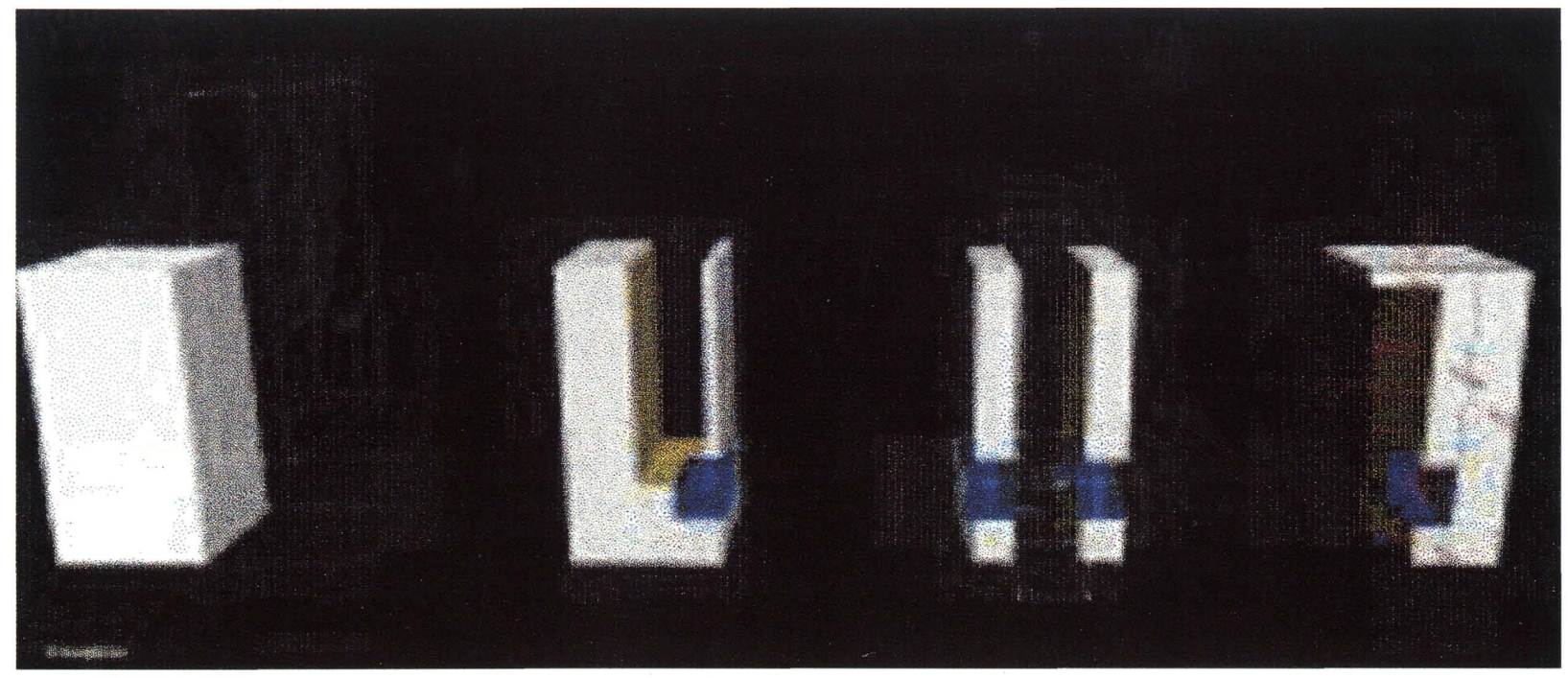

Fig. 2.- La generación de espacios dentro de un volumen se realiza utilizando los volúmenes positivos y negativos, como se muestra en esta secuencia.

El programa permite la especificación de parámetros de los objetos en forma interactiva, como también soporta los modelos con atributos tales como forma, geometría, color, material, etc. Tanto los objetos, como los grupos de objetos y los mundos virtuales, pueden ser cambiados en tiempo real, a la vez que permite el movimiento tanto fuera como dentro del propio modelo.

Actualmente, se están desarrollando en el prototipo "agentes inteligentes" tales como el de sonido, el de estimación de costos y el de navegación, utilizando diferentes posibilidades que ofrecen las técnicas de inteligencia artificial y los nuevos avances experimentados por las interfases usuario-computador, en las cuales no entraremos por no ser el objeto de este trabajo, pero que pueden consultarse en las citas bibliográficas.

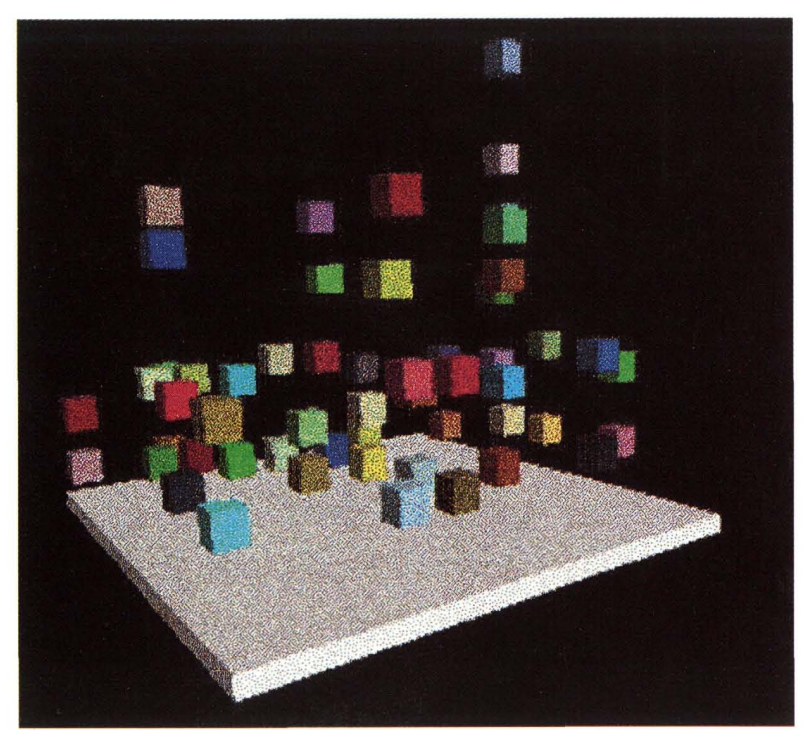

Sculptor no sólo ayuda al usuario en la creación de objetos tridimensionales, sino que, además, es capaz de soportar modelos de comportamiento basados en los principios de la mecánica y de la dinámica. Entre los modelos de comportamiento, caben señalar: la posibilidad de detectar la colisión entre objetos y los efectos de la gravedad sobre los mismos (ver fig. 3), de gran utilidad para el trabajo que aquí se presenta cuando se trata de ver, de forma intuitiva, los efectos que podría causar la eliminación de ciertas partes del modelo, o la modelización de efectos causados por la degradación, etc. Otra posibilidad es la capacidad de movimiento autónomo, bien sea del grupo de objetos o de cada objeto por separado, con ello, es el prototipo quien trabaja por nosotros en la creación de distintas soluciones, si bien la misma resulta de mayor utilidad cuando se trata de generar nuevas propuestas.

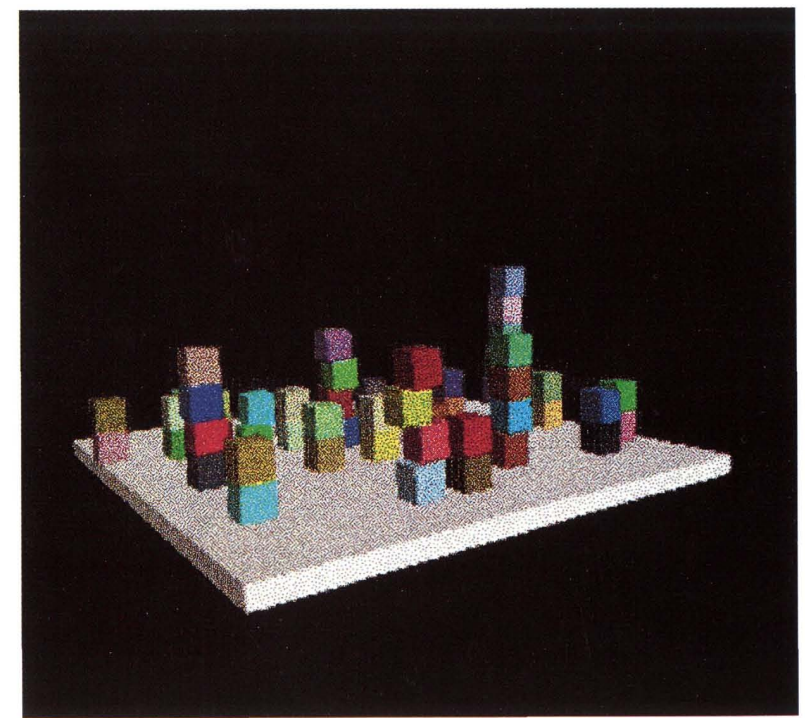

Fig. 3.- Variación del mismo escenario cuando se dota a los objetos de los efectos de gravedad y colisión.

(c) Consejo Superior de Investigaciones Científicas 
Por último, se puede dotar a los objetos con un cierto grado de conocimiento acerca de ellos mismos, de su comportamiento o de su entorno. Esto permite definir objetos inteligentes que poseen unas ciertas cualidades y un cierto comportamiento, que pueden ser, o bien modelos que representan objetos físicos, tales como elementos de un edificio o mobiliario o bien simples objetos con características funcionales 0 de comportamiento. Estas posibilidades son muy útiles en diseño colaborativo, en el cual, los distintos participantes en el proceso de diseño, tienen acceso al mismo escenario y actúan sobre el modelo aplicando modificaciones acordes con su área de experiencia.

\section{EI edificio modelizado: St. Asvatzatzin}

Con el fin de entender el trabajo que se presenta, se ha modelizado un pequeño edificio religioso, la capilla mausoleo dedicada a St. Asvatzatzin (la madre de Dios), perteneciente al monasterio de Amaghu Noravank, situado en la histórica región armenia de Siunik'. Se trata de un complejo que data de finales de la Edad Media, cuando el país tuvo un importante florecimiento artístico [Alpago, 1986], [Mnatsakanyan, 1960], [Cuneo, 1978]. E1 monasterio, residencia espiritual de la familia Oberlyan, príncipes de Vayots-Dzor (ver figura 4), está compuesto esencialmente por tres iglesias orientadas, una al lado de la otra: la capilla mausoleo dedicada a St. Asvatzazin, cercana a la muralla sur y dos pequeñas capillas mausoleo, fuera de las murallas, también orientadas al este.

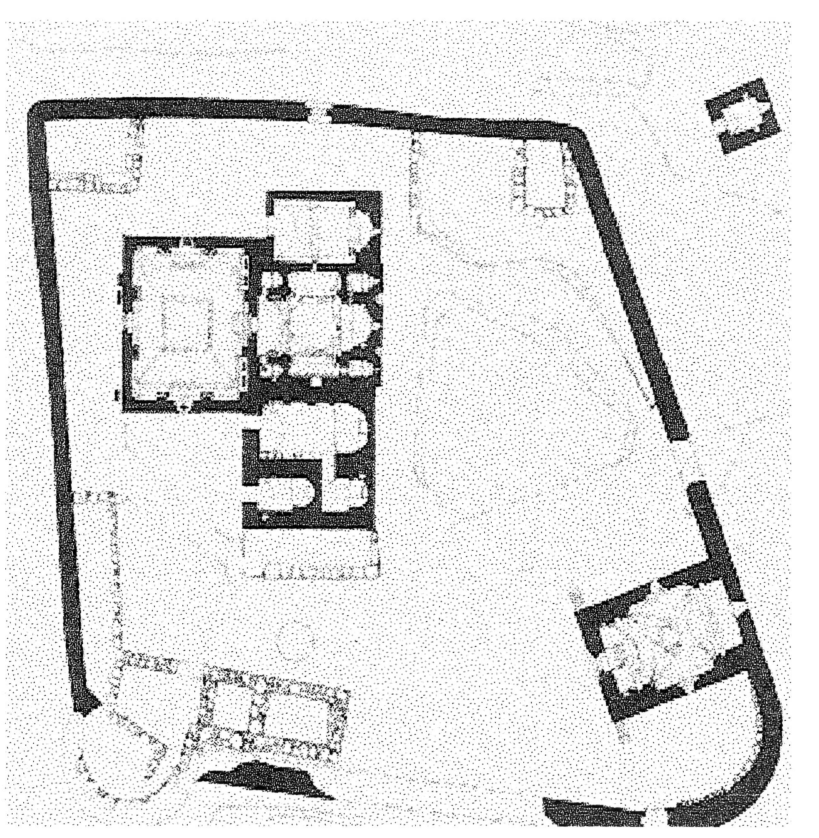

Fig. 5

(c) Consejo Superior de Investigaciones Científicas Licencia Creative Commons 3.0 España (by-nc)
St. Asvatzatzin (1339) es uno de los últimos edificios de este tipo de la alta Edad Media en Armenia y el último realizado en el complejo que constituye el monasterio. Del análisis histórico, se cree que dicha capilla fue un trabajo realizado por el maestro cantero Varpet Momik, quien realizó varios trabajos en la región, creando una verdadera escuela, ya que en uno de los trabajos realizados por Momik, un evangelio de alrededor de 1292, se representa un esquema de dicho edificio.

El edificio consiste en 2 plantas cruciformes (el mausoleo en la planta baja y la capilla en la alta), cuyas superficies, suaves y limpias, se unen en agudas esquinas que enriquecen el volumen; probablemente en el origen, y, basándose en estudios tipológicos de este tipo arquitectónico, el edificio poseía una amplia linterna con 12 columnas. A las cualidades arquitectónicas del edificio debe agregarse la exquisita decoración plástica de su exterior, la elegante articulación de las fachadas, la decoración de la parte superior y los relieves de las lunetas de la entrada, los detalles escultóricos de la bóveda de la habitación inferior, etc.

Los primeros documentos fotográficos del monasterio, que fue abandonado después del sismo de 1840 , son de principios de este siglo [Strzygowsky, 1918], [Hovhannisyan, 1978]. Del examen fotográfico de estas primeras fuentes (fig. 5) puede verse que la linterna estaba totalmente destruida a principios del siglo $\mathrm{XX}$, que la planta superior había sufrido numerosos daños $y$, sus paredes estaban parcialmente colapsadas, 10 que contribuyó a un mayor deterioro del edificio por la falta

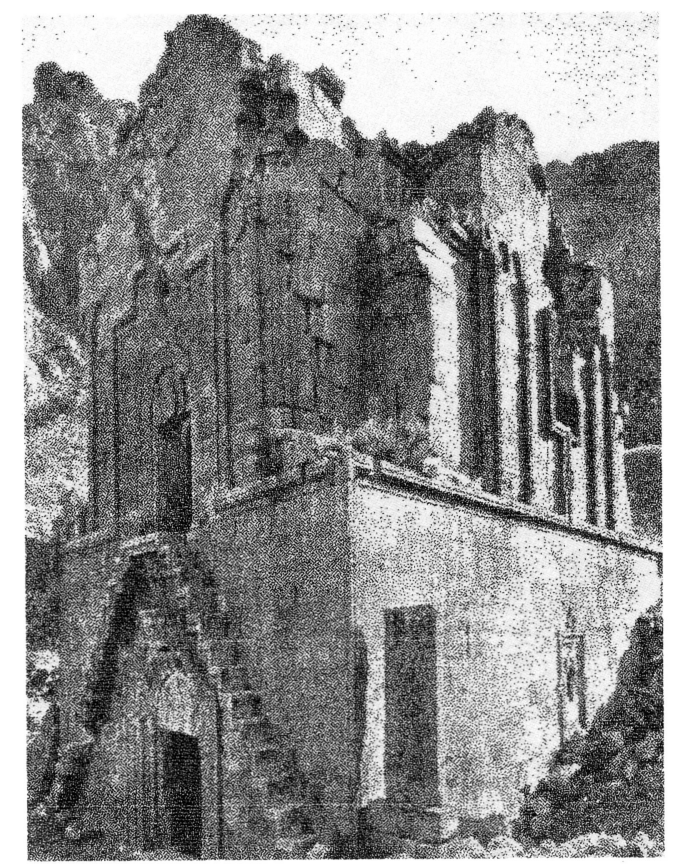

Fig. 4.- Amaghu Noravank.

http://informesdelaconstruccion.revistas.csic.es 
de estos elementos y por la acción climática [Alpago, 1985].

Tras el primer trabajo de restauración (1948-1949), basado en el proyecto de A. Balassanian, el aspecto del edificio (que es el que actualmente presenta) es el que se ve en las figuras $6 a$ y $6 \mathrm{~b}$, con el segundo piso, y en especial la fachada este totalmente reconstruídas, y con un techo provisional de madera y chapas metálicas, con el fin de protegerlo de ulteriores daños causados por agentes climáticos.

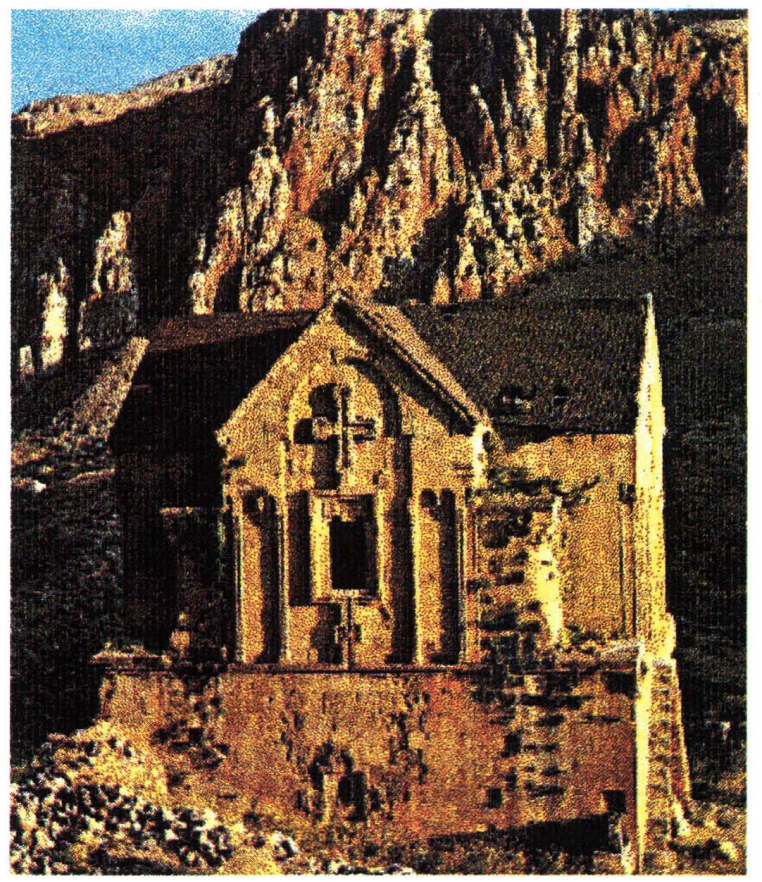

(a)



(b)

Fig. 6

(c) Consejo Superior de Investigaciones Científicas Licencia Creative Commons 3.0 España (by-nc)
Ante la necesidad de realizar nuevos trabajos de restauración, se plantean diferentes propuestas, todas ellas basadas en los actuales criterios sobre restauración y conservación de edificios históricos, que se pueden resumir en [Shahinian, 1993]:

- Mantener el monumento en su estado actual, realizando sólo una intervención que consolide su status quo (por ejemplo: eliminando la construcción del tejado, colocado como protección);

- Reemplazando el actual tejado colocado como protección, por una construcción más estable, quedando, por tanto, el monumento en condiciones similares a las que pueden verse en la figura 6;

- Reconstruyendo las partes inexistentes del edificio (por ejemplo: las bóvedas, el tejado, la linterna y el techo de la misma) de acuerdo con las partes existentes, utilizando para ello materiales originales;

- Reconstruyendo las partes inexistentes del edificio, como se mencionó anteriormente, pero utilizando para su reconstrucción otros materiales (piedra, hormigón armado, acero, etc.);

- Integrando los espacios existentes en soluciones arquitectónicas modernas.

No es el propósito de los autores de este artículo realizar una crítica sobre cada una de las intervenciones que se proponen, sino el discutir sobre lo âpropiado que resulta, o no, el utilizar herramientas CAAD, en especial la que se describe en el punto 2, dentro del contexto del proceso de restauración.

\section{Estudio tipológico del monumento}

Para analizar los distintos escenarios de restauración propuestos para la capilla máusoleo dedicada a St. Asvatzatzin, fue necesario crear una librería de los distintos elementos tipo encontrados en el propio monumento, así como de los extraídos del estudio de distintas tipologías de arquitectura medieval armenia.

Dado que la finalidad buscada utilizando este prototipo era el estudio tipológico, los elementos creados fueron sencillos, pero si por otras razones fuese necesario, el catálogo podría incluir objetos más complejos. El usuario puede seleccionar los mencionados objetos de un menú e insertarlos en el escenario específico (ver fig. 7). 


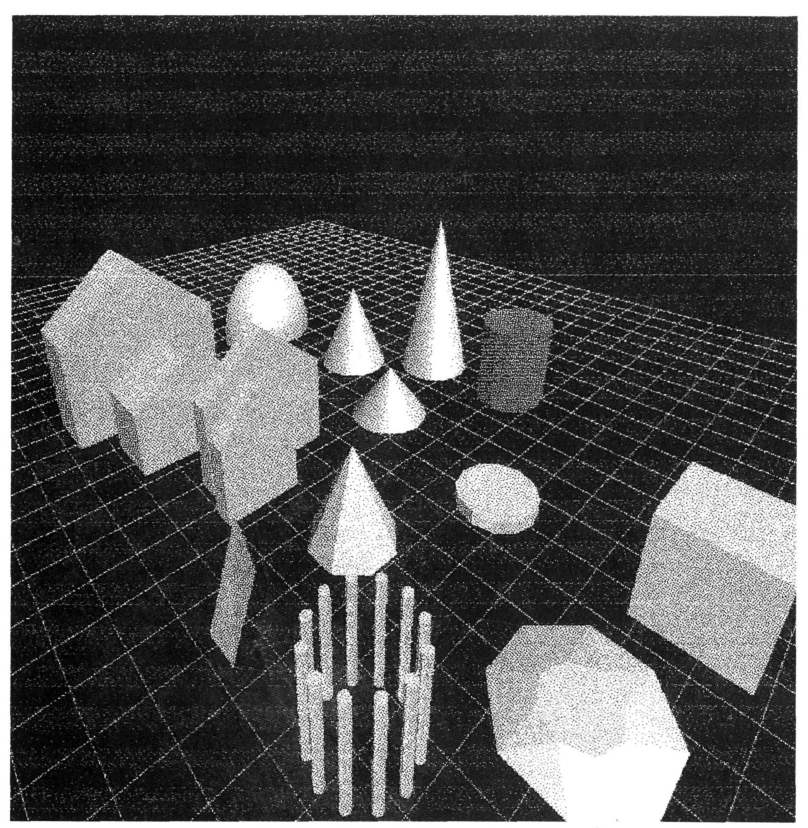

Fig. 7

La subyacente funcionalidad que posee Sculptor permite entender la estructura y su tipología, por ejemplo haciendo una simulación del actual edificio (ver fig. 8) y sus variaciones, ya que con la implementación realizada se pueden generar, a la vez que mover, objetos tales como cubos, cilindros, esferas, etc., de manera muy sencilla, seleccionando los mismos y colocándolos en el espacio donde se pueden rotar, desplazar, o bien cambiar sus dimensiones. Cada cosa "ocurre" instantáneamente y se visualiza al mismo tiempo en la pantalla.

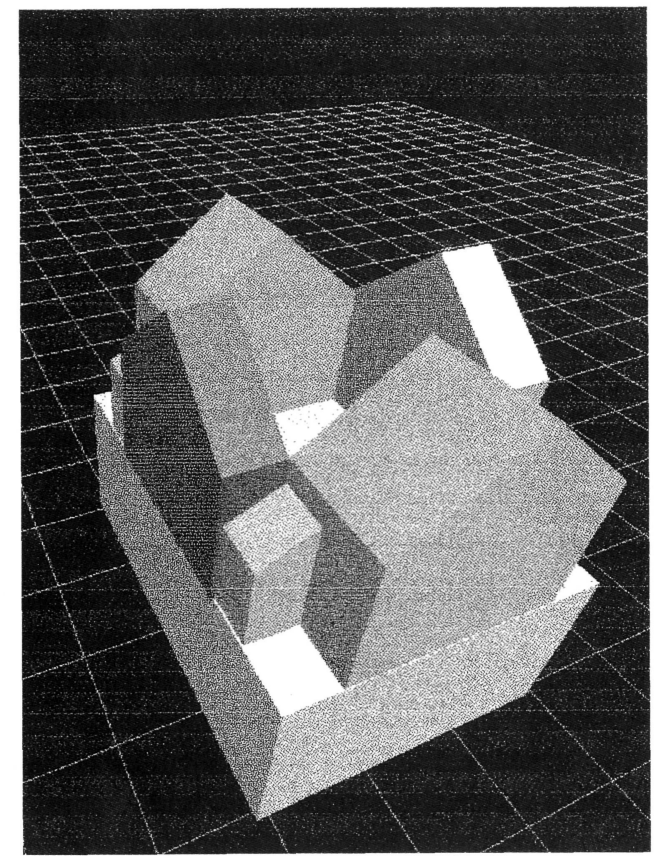

Fig. 8

(c) Consejo Superior de Investigaciones Científicas Licencia Creative Commons 3.0 España (by-nc)
Sculptor soporta funciones que va aprendiendo de los distintos eventos, como si del mundo real se tratara, cuando trabajamos con modelos usando objetos físicos. Esto resulta de gran ayuda para el usuario, ya que se puede definir una gramática -combinaciones válidas de elementos- que facilite la combinación de distintos elementos, siempre y cuando sea posible.

La idea es crear una caja de elementos virtuales que permita, de una forma interactiva y directa, jugar con esos objetos en el ordenador, con el fin de describir los distintos escenarios mencionados anteriormente desde un punto de vista tipológico. La clave de la propuesta es la posibilidad de ver los objetos que se han creado en un entorno virtual y su interacción directa con el modelo. La posibilidad de que los objetos sean inteligentes permite que cada uno de ellos conozca lo necesario sobre sí mismo, así como de su entorno y, por tanto, muestre un comportamiento específico como respuesta a las interacciones del usuario; por ejemplo, un objeto paramétrico cambia su forma de acuerdo con un conjunto de proporciones que se derivan del análisis del edificio que se analiza. De este modo, los modelos creados están determinados por las propiedades que cada elemento tiene definidas y, por tanto, obedecen a unas reglas de composición que previamente han sido prefijadas (ej. una linterna de forma octogonal no puede situarse sobre un tambor de forma hexagonal).

Ciertos comportamientos físicos básicos pueden adjudicarse a cada objeto [Barzel, 1992] y de este modo, ciertos fenómenos naturales como gravedad, colisión, etc. pueden ser, de alguna manera, modelizados. Por ejemplo: la gravedad, es algo que, en sí mismo, no tiene una representación visual, pero si su efecto puede ser visualmente modelizado, es algo fundamental que debe tener cualquier herramienta de modelización arquitectónica. En Sculptor, el efecto de la gravedad puede asignarse a cada objeto o no. Esto provoca que cada objeto caiga al suelo o se apoye sobre otro objeto cuando el efecto de gravedad está activo (fig. 3). Es de gran importancia el uso de la gravedad en los objetos que constituyen el modelo, con el fin de visualizar, en cierta forma, los movimientos de ciertos elementos cuando uno de ellos desaparece (por ejemplo, nos permite ver, de una forma gráfica, el efecto de la desaparición de parte de la estructura causada por un deterioro de la misma, sin necesidad de una modelización exacta del fenómeno que lo ha causado).

Otra posibilidad del prototipo, como ya ha sido mencionado, es la detección de colisiones. Como ocurre en la naturaleza, cuando un objeto sólido choca con otro objeto su movimiento se ve detenido, esto hace que las posibilidades de movilidad de los objetos, o las modificaciones de sus dimensiones, sólo puedan ocurrir dentro de unas condiciones de validez, por ejemplo si un obieto se encuentra con otro $v$ no se trata de una 
intersección entre los mismos, entonces se produce la colisión, resultando imposible realizar cualquier movimiento o cualquier modificación del objeto. Ambos conceptos son importantes cuando se trata de modelizar el mundo real en un entorno virtual.

Para realizar algunos de los modelos que representan el posible aspecto que tenía el monumento en su origen (basándonos en el estudio de las tipologías), es necesario, combinar los elementos simples que se encuentran en la librería básica de elementos, o bien importando los mismos de otros programas y componerlos hasta conseguir el modelo deseado. A título de ejemplo. véase la fig. 9, donde se muestran distintos planteamientos de cómo podía ser la parte inexistente hoy en día del monumento estudiado (ver [Guevorkian, 1985] para mayor información sobre restauración de monumentos en Armenia).
La interacción con el modelo se incrementa cuando se utilizan dispositivos 3D, tanto de entrada como de salida, a la vez que da un mayor realismo a las escenas generadas en el ordenador, ya que el usuario forma parte del propio escenario.

No obstante las posibilidades y ventajas que presenta Sculptor para la realización de los estudios preliminares, es necesario, sin embargo, recurrir a programas comerciales, cuando se trata de reconstruir el monumento completo con mayor nivel de detalle, como el que se muestra en la figura 10. Esta representación puede utilizarse para los trabajos ulteriores que permiten la realización de planos y secciones, así como para representar los complejos detalles constructivos del monumento.

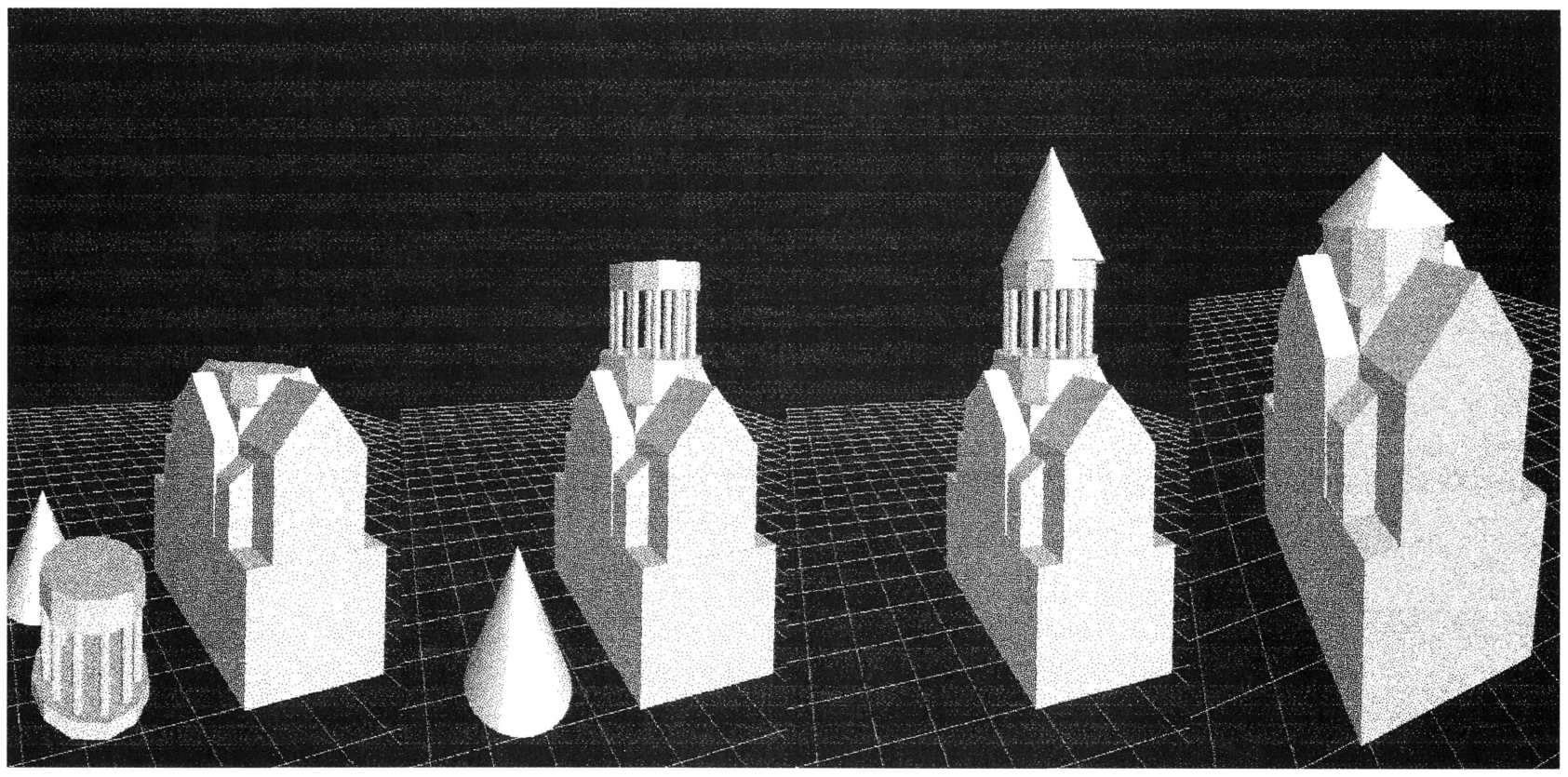

Fig. 9

A diferencia de otras herramientas, Sculptor trabaja con los modelos sólidos como un escultor trabaja para crear una figura en piedra o, en otras palabras, en vez de utilizar las operaciones lógicas entre volúmenes como sustracción, unión, etc., trabaja con volúmenes positivos y negativos. El concepto básico que utiliza, es que siempre un volumen negativo crea un espacio cuando éste intersecta con un volumen positivo (sólido), pero no ocurre nada cuando dos volúmenes negativos se intersectan entre sí. Esta posibilidad es muy útil para poder abrir en los elementos sólidos los huecos que corresponden a ventanas o a puertas, o bien para modelizar el proceso constructivo.

(c) Consejo Superior de Investigaciones Científicas Licencia Creative Commons 3.0 España (by-nc)

\section{Algunos comentarios finales}

Para la creación de modelos virtuales, de gran utilidad para los expertos involucrados en la conservación del patrimonio arquitectónico, se requieren estaciones gráficas potentes y avanzadas, si lo que se busca es crear unambiente interactivo y un escenario realista,aun cuando los modelos sean de mediana complejidad.

Sin embargo, los avances tanto en hardware como en software que se están experimentando en los últimos años, hará posible que estos medios estén disponibles en equipos personales. 

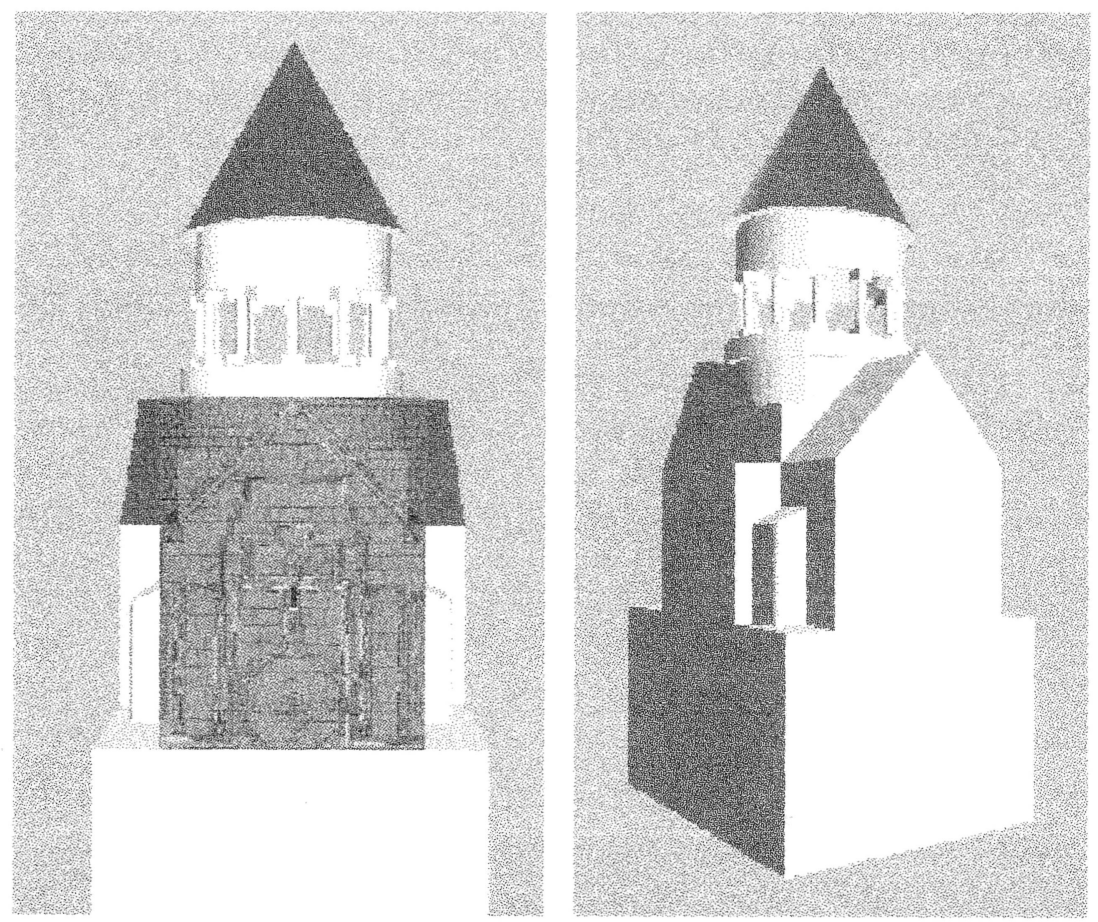

Fig. 10.- Reconstrucción de St. Asvatzatzin [Guevorkian, 1985].

Las técnicas de modelización que se han descrito anteriormente están en su fase inicial, por lo que se necesita seguir investigando, con el fin de desarrollar mejores prototipos que permitan definir un entorno adecuado en el cual, los expertos puedan modelizar sus conceptos e ideas.

Las herramientas que se han descrito, no sólo son adecuadas para la práctica profesional, sino que son adecuadas desde el punto de vista docente para la formación de los futuros profesionales. Los diferentes profesionales que intervienen en la restauración de monumentos necesitan tener experiencia en el uso de este tipo de herramientas, que les pueden ayudar a visualizar y contrastar sus ideas antes de afrontar la restauración del monumento.

Las personas que trabajan en la restauración de monumentos, no sólo deben ser capaces de entender el significado social de los mismos, los aspectos tecnológicos y la naturaleza de sus materiales, sino que también deben ser capaces de adecuar los espacios de acuerdo con el futuro uso y funciones del edificio. Para ello deben tener una visualización clara de las soluciones planteadas a problemas complejos como son, en general, los que se manejan cuando se trata de cambiar las funciones de un edificio histórico, para adaptarlo a su futuro uso, tratando de hacer modificaciones mínimas en el mismo. En otras palabras, deben ser capaces de proyectar cualquier modificación que permita adaptar el edificio a sus nuevas funciones, pero manteniendo los rasgos esenciales del mismo [COTAC, 1994]. El poder trabajar en entornos virtuales, con el fin de comprobar el impacto de las distintas propuestas, así como ver el aspecto final del edificio antes de abordar la propia restauración, son esenciales al respecto.

Si bien son varios los aspectos que deben mejorarse en las herramientas CAAD como la que se presenta en este trabajo, y por ello se sigue investigando al respecto, no obstante, es conveniente ponerlas en conocimiento de la comunidad científica, con el fin de crear herramientas adecuadas a los fines que se persiguen.

\section{Información adicional}

Se puede encontrar información adicional del tema en las siguientes páginas-www:

Sobre Sculptor:

http://caad.arch.ethz.ch/ kurmann/sculptor.html

Sobre Armenia:

http://caad.arch.ethz.ch/ moreno/armenia.html http://www.darmstadt.gmd.de/ baloian/armenia.html 
BIBLIOGRAFÍA

ALPAGO-NOVELLO, ADRIANO (1985). Amaghu Noravank - documenti di architettura armena. Edizioni Ares, Milano.

ALPAGO-NOVELLO, ADRIANO, ET AL. (1986). Gli Armeni. Arts et cultures. Jaca Book, Milano.

BARZEL, R. (1992). Physical-Based Modelling for Computer Graphics. Academic Press.

COTAC; Conference on Training in Architectural Conservation, (1994). Multi-Disciplinary Collaboration in Conservation Projects in the UK, Based on ICOMOS Guidelines for Education and Training in the Conservation of Monuments, Ensembles and Sites.

CUNEO, P. (1978). Le scuole regionali nell'architettura armena. In Atti del Primo Simposio Internazionale di Arte Armena. Venezia.

GUEVORKIAN, TELMAN (1981). Le système des mesures de l'Armenie medievale et la méthode de son utilisation dans la pratique de la restauration moderne.

KALAWSKY, R. S. (1993). The science of VR and Virtual Environments. Addison-Wesley.
KURMANN, D. (1995). Sculptor - Towards a Computer Tool for Intuitive Design. CEMCO '95, Seminario S1, (Instituto Eduardo Torroja, Madrid), pp. 41-48.

KURMANN, D. \& ENGELI, M. (1996). Modelling Virtual Space in Architecture. VRST '96 - Virtual Reality Software and Technology, M. Green, K. Fairchild and M. Zyda (Eds.), ACM , P. 77-82, Hongkong.

HOVHANNISYAN, K. (1978). Il restauro dei monumenti in Armenia sovietica. Erevan.

MNATSAKANYAN, S. (1981) 'Noravank', Guida in armeno e russo. Erevan.

SCHMITT G. (1994). Scene Animation using Intelligent Objects in a Virtual Design Environment. Speedup Journal, (CSCS, Manno, Switzerland):8/1,pp. 14-20

SHAHINIAN, SARKIS. (1993) S. Asdvadzadzin Burtelashen, Proposta di restauro della cappella funeraria di Amaghu Noravank. Ricerca finale relativa ai corsi elettivi di Restauro e Conservazione dei Monumenti - Strutture Portanti Piane, ETH Zürich.

STRZYGOWSKI, J. (1918). Die Baukunst der Armenier und Europa. Kulturhistorisches Institut der Universitaet Wien, Schroll, Wien.

\section{Publicación del Instituto Eduardo Torroja - CSIC}

\section{JORNADAS SOBRE CEMENTO ALUMINOSO}

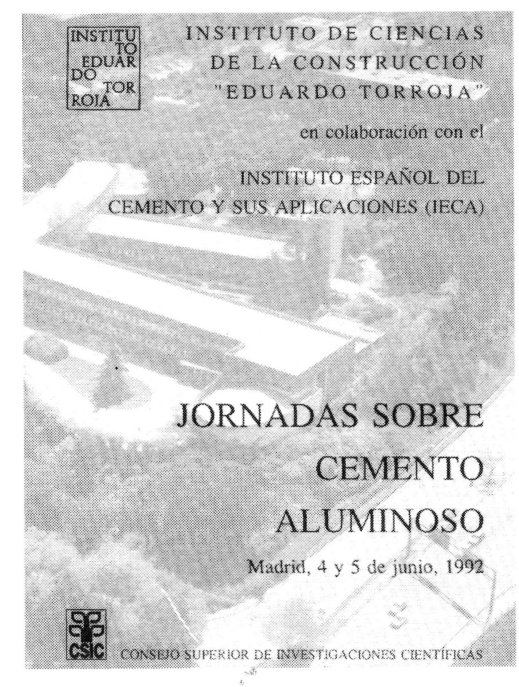

El Instituto Eduardo Torroja publica íntegramente -en colaboración con el Instituto Español del Cemento y sus Aplicaciones (IECA) - las "Jornadas sobre cemento aluminoso", celebradas en junio de 1992.

Se recogen las conferencias dictadas por destacados especialistas en la materia, así como las intervenciones suscitadas en los coloquios y en la interesante Mesa Redonda, tras la cual se clausuraron las Jornadas.

El tratamiento dado al téma del hormigón y cemento aluminoso por los intervinientes abarca tanto aspectos rigurosos científico-técnicos como de índole práctica, abordando temas de corrosión de armaduras, durabilidad, protección y reparación de los elementos constructivos con daños causados por diversas motivaciones ampliamente expuestas.

Este libro será, sin duda, una eficaz ayuda tanto para el científico o el técnico estudioso de esta materia, como para aquellos profesionales interesados en un tema de tanta importancia y actualidad como es el relacionado con los hormigones de cemento aluminoso.

Un volumen de $15,5 \times 22,5 \mathrm{~cm}, 186$ págs., con figuras de línea, gráficos, tablas, etc. 